\title{
Wie sich COVID-19 in der 3D-Zellkultur simulieren lässt
}

DORIS WILFLINGSEDER, WILFRIED POSCH

INSTITUT FÜR HYGIENE UND MEDIZINISCHE MIKROBIOLOGIE, MEDIZINISCHE UNIVERSITÄT INNSBRUCK, ÖSTERREICH

\section{Excessive inflammation triggered by a hitherto undescribed mecha- nism is a hallmark of severe SARS-CoV-2 infection and is associated with enhanced pathogenicity and mortality. Complement hyper activa- tion promotes lung injury and was observed in patients suffering from MERS-CoV, SARS-CoV-1 and SARS-CoV-2 infections. To evaluate the very first interactions of SARS-CoV-2 patient isolates with human epi- thelial tissues, 3D models of the human respiratory tract as well as lung organoids are highly suitable.}

DOI: $10.1007 / \mathrm{s} 12268-022-1712-y$

(C) Die Autorinnen und Autoren 2022

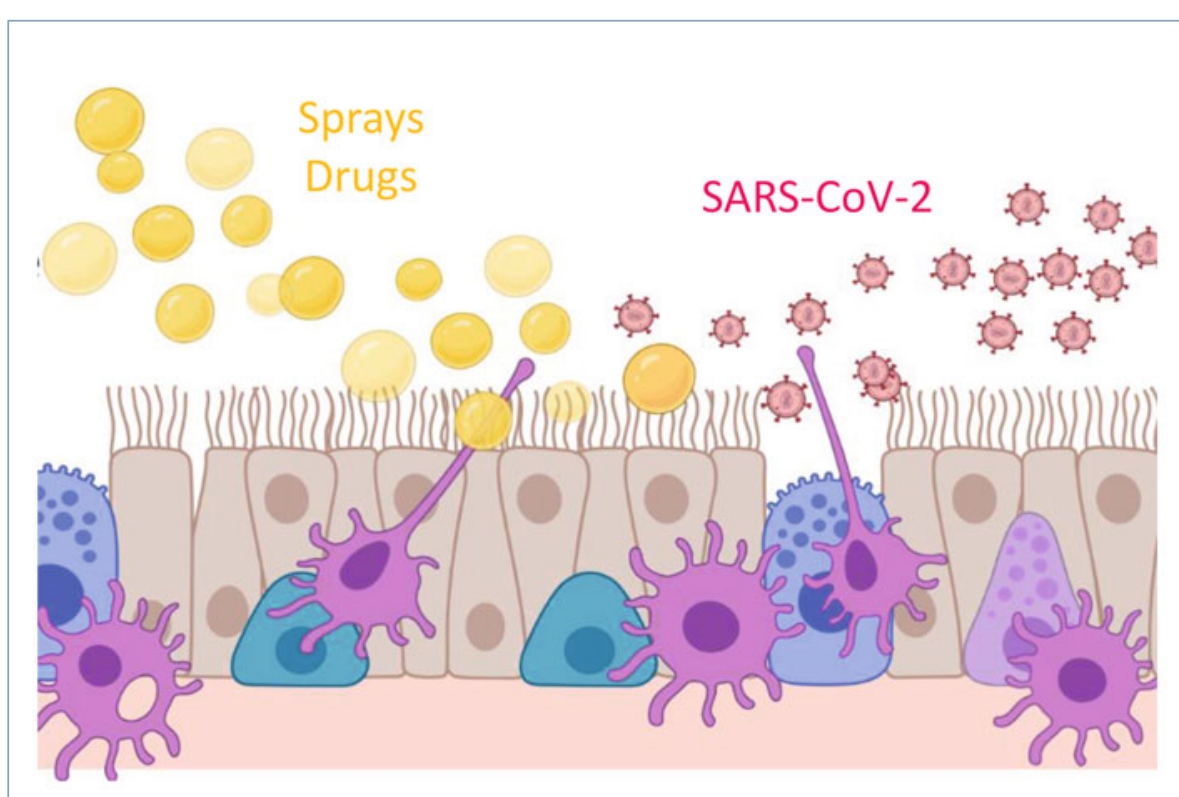

A Abb. 1: Untersuchung von COVID-19 im standardisierten 3D-Zellkulturmodell. Das Barrieremodell des respiratorischen Trakts besteht aus hoch differenziertem Flimmerepithel (braun) sowie mukusproduzierenden Zellen (blau, violett). Das pseudostratifizierte Luft-Flüssig-Interphase-Modell kann durch Stammzellen im System (türkis) erneuert werden, sodass das Zellkulturmodell sich auch für Langzeitversuche eignet - es kann bis zu 2 Jahren in Kultur gehalten werden. Die Epithelien können nach Differenzierung von der apikalen (Luft-)Seite mit SARS-CoV-2 infiziert werden, um die Situation im Körper möglichst naturgetreu nachzustellen. Des Weiteren können die Gewebe mit antiviralen Sprays von der apikalen Seite her behandelt werden und deren Wirkung gegen SARS-CoV-2 getestet werden. Immunzellen von Interesse (hier dendritische Zellen) können bei Bedarf leicht in das 3D-Zellkultursystem integriert und so auch zusätzliche Faktoren, die im Körper eine Rolle während COVID-19 spielen, analysiert werden. Erstellt mit BioRender.
Frühe Ereignisse, die direkt nach der Übertragung von SARS-CoV-2 auf Gewebe des Atmungstrakts auftreten, bestimmen den Verlauf der Erkrankung maßgeblich. Bei einigen Patienten führt eine Infektion mit COVID-19 zu einer überschießenden Immunantwort und damit verbunden der Erzeugung eines proinflammatorischen Milieus. Die Entwicklung eines Zytokinsturms und eine akute Lungenschädigung (ARDS, acute respiratory distress syndrome) sind unerwünschte Folgen von COVID-19 und führen zu einer erhöhten Morbidität und Mortalität durch Gewebsverletzungen sowie Multiorganversagen [1-6].

Mit neuartigen 3D-Zellkultursystemen lassen sich Wechselwirkungen zwischen Krankheitserregern und immunkompetenten Barrieremodellen simulieren und studieren, was auch Angriffsflächen für neue Therapieoptionen möglich macht (Abb. 1). Daher spielt auf der Suche nach neuen Medikamenten und Therapien auch die Grundlagenforschung eine wesentliche Rolle und besonders innovative, vielversprechende und tierversuchsfreie Ansätze können das Verständnis von ersten SARS-CoV-2-Interaktionen an den Atemwegsepithelien verbessern.

Das gesamte respiratorische Epithel von der Nasenhöhle bis hinab zu den Alveolen ist konstant Krankheitserregern ausgesetzt. Wenn die Krankheitserreger in der Lage sind, das mukusproduzierende, pseudostratifizierte Flimmerepithel zu durchbrechen, muss spontan die angeborene Immunantwort aktiviert werden. In diesem Kontext wurde gezeigt, dass die Flimmerepithelien, die eigentlich nicht zu unserem Immunsystem gehören, Proteine des Komplementsystems produzieren und sezernieren können - eine sofortige Immunreaktion unseres Körpers [7]. Auch in schwer erkrankten COVID-19-Patienten wurde beschrieben, dass Komplement bei der Verstärkung der Entzündungsreaktion eine wichtige Rolle spielen könnte. Das Komplementsystem, das im Normalfall vor Virusinfektionen schützen soll, könnte somit während schwerer COVID-19-Verläufe fatal wirken und die Gewebszerstörung vor- 


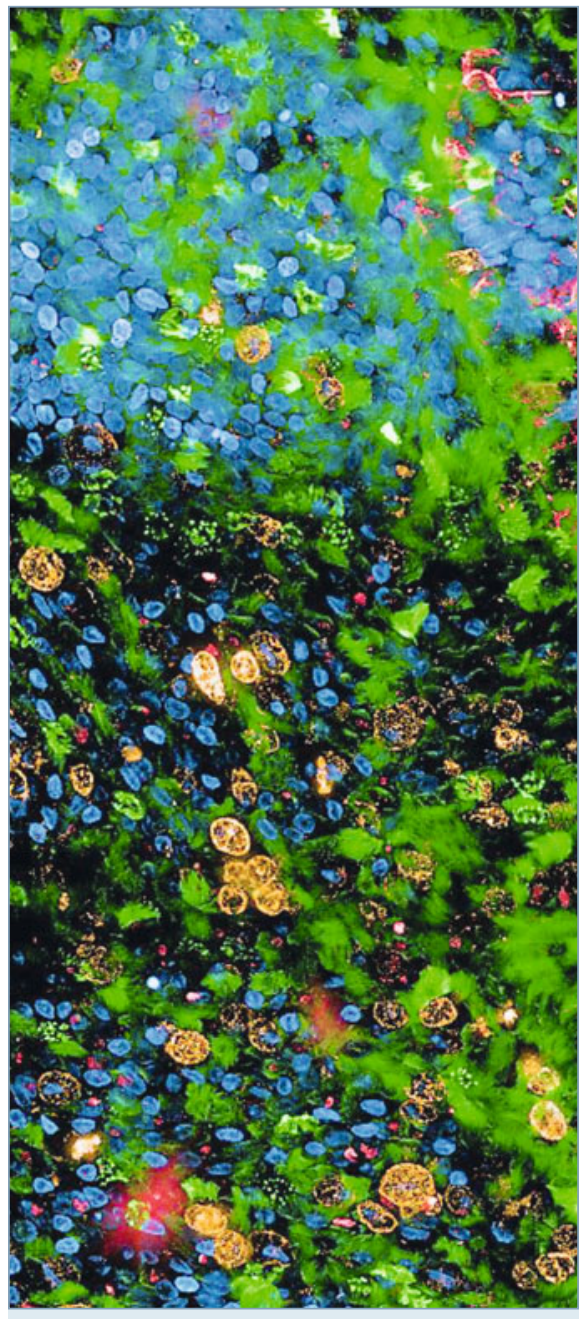

$\triangle$ Abb. 2: SARS-CoV-2 zerstört die Gewebeintegrität und verursacht Mukushypersekretion im 3D-Barrieremodell. Nach 2 Tagen Infektion mit einer hohen Viruslast eines SARS-CoV-2-Patientenisolats (rot) sieht man bereits eine massive Zerstörung des Gewebeverbands, erkennbar an fragmentierten Zellkernen (Nuklei, blau) und Löchern in den Geweben. Des Weiteren sieht man eine Mukusüberproduktion (orange) in infizierten Epithelien. Es werden sowohl Flimmerepithelzellen (grün) als auch mukusproduzierende Zellen (orange) durch das SARSCoV-2-Wildtyp-Virus, welches zu Beginn der Pandemie isoliert wurde, infiziert.

antreiben, wenn das Flimmerepithel durch lokale Komplement- und damit verbunden Anaphylatoxinproduktion die Entzündungsreaktion verschlimmert.

Durch Simulierung einer akuten SARSCoV-2-Infektion in einem über die letzten Jahre optimierten und standardisierten LuftFlüssig-Modell des oberen und unteren respiratorischen Trakts $[8,9]$ sowie in Lungenorganoiden, kann quasi im Livemodus beobachtet werden, wie Epithelzellen auf die Infektion reagieren, welche Zytokine und
Anaphylatoxine ausgeschüttet werden, wie schnell Viruspartikel weitertransportiert werden, beziehungsweise welche Gewebszerstörung das Virus verursacht. Dies sind nur einige der vielen Analysen, die in der 3D-Zellkultur durchgeführt werden können. Interessant ist auch die Behandlung solcher Kulturen mit antiviralen oder nicht konventionellen, aber vielversprechenden, Medikamenten, wie z. B. Komplementblockern. Unter Verwendung der 3D-Zellkulturmodelle wurden folgende Fragestellungen im Jahr 2020 sofort nach Auftreten von SARS-CoV-2 in Europa und Expansion sowie Charakterisierung von Virusisolaten aus Patienten bearbeitet.

\section{Was passiert direkt nach SARS-CoV-2- Übertragung an den epithelialen Barrieren?}

Hoch differenzierte, schleimproduzierende Flimmerepithelien des oberen und unteren Respirationstrakts wurden mit SARSCoV-2-Patientenisolaten infiziert und nach zwei Tagen mittels Immunfluoreszenz analysiert. Die Gewebemodelle zeigten eine starke Infektion mit SARS-CoV-2 unabhängig vom Zelltyp - sowohl Wimper- als auch mukusproduzierende Zellen wurden von SARSCoV-2 infiziert (Abb. 2, [10]). Die Gewebe wurden signifikant zerstört, wie man anhand von fragmentierten Zellkernen erkennen konnte [10]. Des Weiteren fiel in den infizierten Gewebemodellen sofort eine Hypersekretion von Schleim sowie Schleimpfropfenbildung auf. Im Gegensatz dazu waren in nicht infizierten, scheinbehandelten Kontrollen, Mukuspfropfen nicht nachweisbar [10]. Bei der Analyse schwerkranker COVID-19-Patienten mit Atemwegsobstruktion und Atemversagen wurden sowohl Mukuspfropfenbildung als auch -hypersekretion in Kombination mit einer Hyperaktivierung proinflammatorischer Zytokine beschrieben [11]. Diese Veränderungen zusammen mit einer reduzierten mukoziliären Clearance, die ebenfalls in unseren infizierten 3D-Modellen nachgewiesen wurde, bilden einen Teufelskreis, der zur - während COVID-19 beobachteten Gewebszerstörung führt.

\section{Welche Mechanismen des angeborenen Immunsystems werden nach SARS-CoV-2-Infektion von humanen respiratorischen Geweben aktiviert?}

In einem nächsten Schritt wurden die immunologischen Antworten auf die SARS-
CoV-2-Infektion, die von den - nicht zum Immunsystem gehörenden - Epithelzellen produziert und sezerniert wurden, untersucht [12]. Da Komplementhyperaktivierung Lungenschädigung verursacht und nicht nur in Patienten, die an MERS-CoV und SARSCoV-1 erkrankt waren, sondern auch bei SARS-CoV-2-infizierten Individuen festgestellt wurde, studierten wir lokale Komplementproduktion in 3D-Zellkulturmodellen, die mit SARS-CoV-2 infiziert wurden [12]. Nach Infektion wurden Viruslast, Infektiosität der neu produzierten Viruspartikel, intrazelluläre Komplementaktivierung, Entzündungsmechanismen und Gewebszerstörung mittels quantitativer RT-PCR, Hochdurchsatzscreening-Analysen, Plaque-Assays, Luminex-Analysen und Erfassung des transepithelialen Widerstands gemessen [12]. Quantitative Analysen all dieser Parameter sind in Abbildung 3 dargestellt. Im SARS-CoV-2-infizierten 3D-Zellkulturmodell konnte bei starker Infektion eine vollständige Zerstörung der epithelialen Integrität beobachtet werden, die mit einer verstärkten Produktion der Komplementkomponente 3 (C3), einer Bildung des Membranangriffskomplexes (MAC) sowie einer Ablösung von Zellen aus dem Gewebeverband und einer Freisetzung des Virus in die Überstände verbunden war. SARSCoV-2-Infektion wurde nicht nur an der oberen Epithelschicht nachgewiesen, sondern die Viruspartikel drangen tief in das pseudostratifizierte Epithel ein. Dies ging einher mit einer C3-Aktivierung und auch C3-Ablagerung auf toten oder beschädigten Zellen. Neben einer signifikant höheren C3-Aktivierung in SARS-CoV-2-infizierten Zellen des Respirationstrakts wurden auch signifikant höhere Konzentrationen des Anaphylatoxins C3a desArg sowie den proinflammatorischen Zytokinen Interleukin(IL)-6, Monozytenchemotaktisches Protein-1 (MCP-1), IL-1 $\alpha$ und RANTES nachgewiesen [12]. Die Komplementaktivierung zu stoppen war somit ein logisches, nächstes Vorgehen, um die SARS-CoV-2-induzierten Entzündungsreaktionen und die Gewebszerstörung zu blockieren. Daher wurden Antagonisten der Anaphylatoxinrezeptoren $\mathrm{C} 3 \mathrm{aR}$ und $\mathrm{C} 5 \mathrm{aR}$ verwendet und deren Effekt mit oben genannten Methoden analysiert. Die Rezeptoren wurden sowohl prophylaktisch als auch einige Stunden nach erfolgter SARSCoV-2-Infektion zugegeben. Unabhängig vom Zugabemodus konnte gesehen werden, dass die Blockierung von C3aR die virale 
Infektion und die Entzündungsreaktion in den Atemwegsgeweben verringerte. Einen größeren Effekt hatte jedoch der C5aR-Antagonismus - Blockierung dieses Rezeptors auf der basolateralen, also der Körperinnenseite zugewandten, Seite stellte die Gewebsintegrität vollständig wieder her, regulierte die lokale Komplement-C3-Produktion signifikant herunter und brachte damit verbunden die C3a- und proinflammatorischen Spiegel wieder auf den Normalwert zurück. Es wurde auch gezeigt, dass C5aR-Antagonismus mit niedriger Viruslast korreliert [12].

Unsere Ergebnisse weisen auf eine neuartige therapeutische Interventionsstrategie hin, bei der Hochrisikopatienten in einem frühen Stadium des Krankheitsverlaufs Komplementinhibitoren verabreicht werden, um eine übermäßige Entzündungsreaktion und die damit verbundenen pathologischen Folgen zu verhindern. Verschiedene Komplementinhibitoren befinden sich derzeit in fortgeschrittenen Stadien klinischer
SARS-CoV-2-Studien (z. B. AMY-101, Eculizumab, Ravulizumab) und könnten über Vernebler in die Lunge verabreicht werden [13].

Wie kann der Eintritt von SARS-CoV-2 ins Nasen-/Bronchialepithel verhindert werden?

Um die Virusübertragung und die damit verbundene Gewebszerstörung und Komplementhyperaktivierung zu verhindern, sind sofortige Interventionen nötig - daher wurde im 3D-Zellkulturmodell der Nase und des oberen Respirationstrakts ein antivirales Mundspray (ColdZyme/ViruProtect) ausgetestet [14]. Die Anwendung von ColdZyme auf vollständig differenziertes, polarisiertes menschliches Epithel, das an einer LuftFlüssigkeits-Interphase (ALI) kultiviert wurde, blockierte die Bindung von SARS-CoV-2 vollständig wie auch die lokale KomplementC3-Produktion und die damit verbundene Infektion und Zerstörung des Gewebemodells.
Wie bereits oben beschrieben, verursachte die SARS-CoV-2-Infektion ohne vorherige Behandlung mit ColdZyme eine Komplementhyperaktivierung einhergehend mit einer überschießenden Entzündungsreaktion und hoher Virusproduktion. Durch einmalige Vorbehandlung der Gewebe mit ColdZymeMundspray wurden diese Effekte vermieden und so der Transfer von SARS-CoV-2 verhindert [14]. Unsere in vitro-Daten deuten darauf hin, dass ColdZyme-Mundspray einen Einfluss auf die Prävention von COVID-19 haben kann und dass es wichtig ist, bereits zugelassene, antivirale Medikamente auf ihre Wirksamkeit gegen SARS-CoV-2 zu überprüfen.

Humane 3D-Zellkultursysteme eignen sich - wie wir zeigen konnten - nicht nur für die Untersuchung von ersten Wirt-PathogenInteraktionen und Virusdynamiken, sondern bieten auch einen Mehrwert vor allem in der präklinischen Phase für die Testung der Wirkung neuer und innovativer therapeutischer oder umfunktionierter (repurposed drugs) Interventionen.

\section{Hier steht eine Anzeige.}

\section{望 Springer}




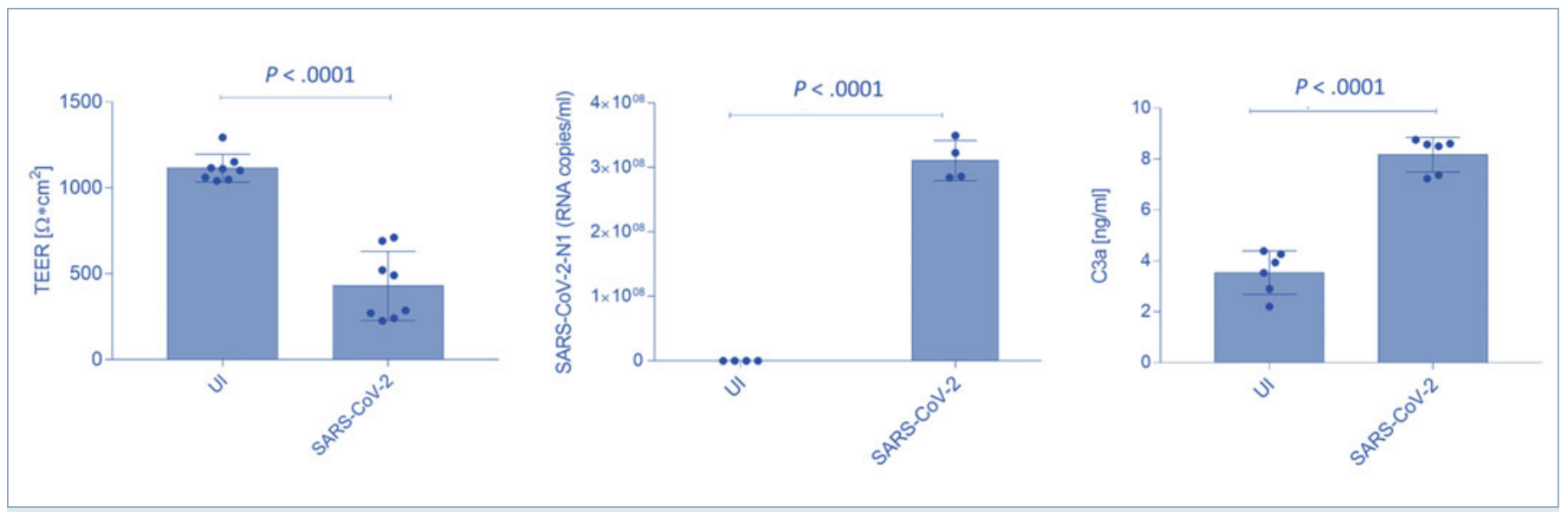

$\triangle$ Abb. 3: Quantitative Analysen der Gewebeintegrität, der Viruslast und Anaphylatoxinproduktion. Links: Nach 3 Tagen Infektion mit einem SARSCoV-2-Patientenisolat sieht man bereits eine massive Zerstörung des Gewebeverbands, wie durch Messung des transepithelialen elektrischen Widerstandes (TEER, transepithelial electrical resistance) detektiert wurde. Ein hoch signifikanter Abfall $(P<0,0001)$ der TEER-Werte ist im SARS-CoV-2infizierten verglichen mit nicht infiziertem Kontrollgewebe (UI) erkennbar. Mitte: SARS-CoV-2-Virus wurde in hoher Konzentration an Tag 3 nach Infektion von ALI-Zellkulturmodellen im basolateralen Überstand mittels RT-PCR und absoluter Quantifizierung analysiert, während in nicht infizierten Kontrollgeweben - wie erwartet - kein Virus nachweisbar war. Rechts: Signifikant höhere Freisetzung $(P<0,0001)$ des Anaphylatoxins $C 3 a$ wurde in SARS-CoV-2-infizierten versus nicht infizierten Geweben detektiert. Signifikante Unterschiede wurden mittels unpaired Student's t-Test in der GraphPad Prism Software ermittelt. Abbildung aus [12], CC BY 4.0.

\section{Literatur}

[1] Magro G (2020) COVID-19: review on latest available drugs and therapies against SARS-CoV-2. Coagulation and inflammation cross-talking. Virus Res 286: 198070

[2] Zhu N, Zhang D, Wang W et al. (2020) A novel coronavirus from patients with pneumonia in China, 2019. N Engl J Med 382: 727-733

[3] Tang N, Li D, Wang X, Sun Z (2020) Abnormal coagulation parameters are associated with poor prognosis in patients with novel coronavirus pneumonia. J Thromb Haemost 18: 844-847

[4] Wang D, Hu B, Hu C et al. (2020) Clinical characteristics of 138 hospitalized patients with 2019 novel coronavirus-infected pneumonia in Wuhan, China. JAMA 323: 1061-1069 [5] Chen G, Wu D, Guo W et al. (2020) Clinical and immunologic features in severe and moderate coronavirus disease 2019. J Clin Invest 130: 2620-2629

[6] Huang C, Wang Y, Li X et al. (2020) Clinical features of patients infected with 2019 novel coronavirus in Wuhan, China. Lancet 395: 497-506

[7] Gu H, Fisher AJ, Mickler EA et al. (2016) Contribution of the anaphylatoxin receptors, $\mathrm{C} 3 \mathrm{aR}$ and $\mathrm{C} 5 \mathrm{aR}$, to the pathogenesis of pulmonary fibrosis. FASEB J 30: 2336-2350 [8] Zaderer V, Hermann M, Lass-Florl C et al. (2019) Turning the world upside-down in cellulose for improved culturing and imaging of respiratory challenges within a human 3D model. Cells 8: 1292

[9] Chandorkar P, Posch W, Zaderer V et al. (2017) Fasttrack development of an in vitro 3D lung/immune cell model to study Aspergillus infections. Sci Rep 7: 11644

[10] Posch W, Lass-Flörl C, Wilflingseder D (2021) SARSCoV-2-infected primary human airway epithelia illustrate mucus hypersecretion J Allergy Clin Immunol 148: 909 [11] Khan MA, Khan ZA, Charles M et al. (2021) Cytokine storm and mucus hypersecretion in COVID-19: review of mechanisms. J Inflamm Res 14: 175-189

[12] Posch W, Vosper J, Noureen A et al. (2021) C5aR inhibition of nonimmune cells suppresses inflammation and maintains epithelial integrity in SARS-CoV-2-infected primary human airway epithelia. J Allergy Clin Immunol 147: 20832097.e6

[13] Jodele S, Köhl J (2021) Tackling COVID-19 infection through complement-targeted immunotherapy. $\mathrm{Br}$ Pharmacol 178: 2832-2848

[14] Posch W, Vosper J, Zaderer V et al. (2021) ColdZyme maintains integrity in SARS-CoV-2-infected airway epithelia. mBio 12: e00904-21

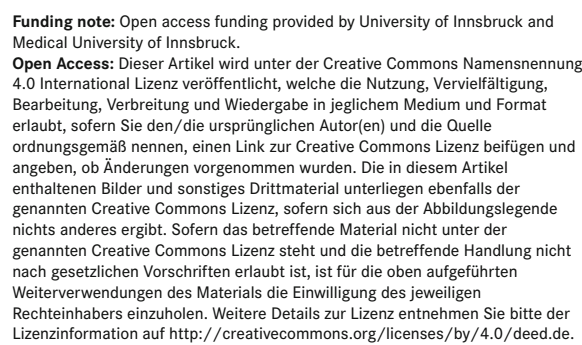

Funding note: Open access funding provided by University of Innsbruck and Medical University of Innsbruck.

Accer 4.0 International Lizenz veröffentlicht, welche die Nutzung, Vervielfältigung, Bearbeitung Verbreitung und Wiedergabe in jeglichem Medium und Form erlaubt, sofern Sie den/die ursprünglichen Autor(en) und die Quelle ordnungsgemäß nennen, einen Link zur Creative Commons Lizenz beifügen und angeben, ob Änderungen vorgenommen wurden. Die in diesem Artikel enthaltenen Bilder und sonstiges Drittmaterial unterliegen ebenfalls der genannten Creative Commons Lizenz, sofern sich aus der Abbildungslegen nichts anderes ergibt. Sofern das betreffende Material nicht unter der Wench Weiterverwendungen des Materials die Einwilligung des jerf Rechteinhabers einzuholen. Weitere Details zur Lizenz entnehmen Sie bitte der Lizenzinformation auf http:// /creativecommons.org/licenses/by/4.0/deed.de.

Korrespondenzadresse:

Prof. Dr. Doris Wilflingseder

Institut für Hygiene und

Medizinische Mikrobiologie

Medizinische Universität Innsbruck

Schöpfstraße 41/R311

A-6020 Innsbruck

doris.wilflingseder@i-med.ac.at

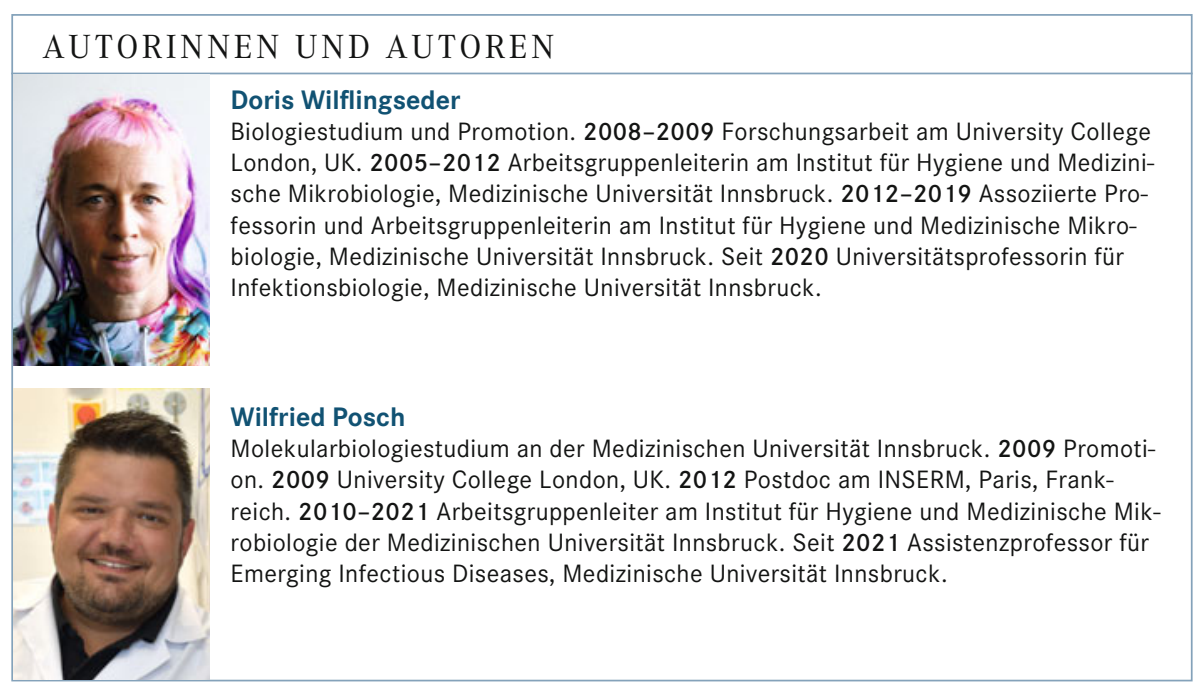

